\title{
Occupational Malignant Neoplasm
}

National Cancer Institute

\section{Source}

National Cancer Institute. Occupational Malignant Neoplasm. NCI Thesaurus. Code C4993.

Malignant neoplasm secondary to occupational exposure to carcinogens. 